\title{
Prevención y control de desórdenes de la voz en los docentes
}

*Elsa Patricia Morales Pérez

\section{RESUMEN}

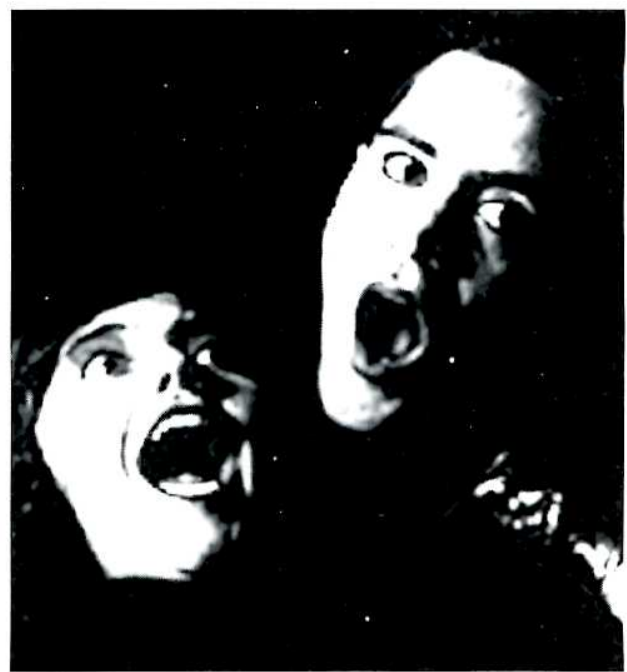

La presencia de problemas en la voz es frecuente entre docentes, debido a que ellos requieren para el desempeño de sus funciones un uso vocal continúo y la mayoría no han recibido información ni instrucción sobre la voz y el uso adecuado de la misma. Estos problemas se pueden prevenir si se realiza un efectivo control de los riesgos ocupacionales que pueden desencadenar desórdenes de la voz. El control de estos riesgos es responsabilidad de docentes, instituciones educativas a cargo de su formación, empleadores y servicios de salud, y cada uno de estos grupos debe tomar conciencia sobre la importancia. En este artículo se resumen los principales factores de riesgo para los profesores y las repercusiones que éstos tienen sobre la voz; proporciona información sobre cómo detectar los primeros síntomas de un problema de voz y estar alerta para controlarlos a tiempo. La información presentada permite conocer las principales áreas de trabajo para llegar a optimizar el acto vocal y proporciona recomendaciones prácticas para los cuidados de la voz.

PALABRAS CLAVE: Voz, fonación, promoción en salud, calidad de la voz.

¡Nadie sabe lo que tiene hasta que lo pierde! (Refrán popular)

\section{INTRODUCCIÓN}

Iniciar el tema con este refrán popular tiene por objetivo invitarlos a reflexionar sobre su contenido aplicado a la voz. La voz es algo que acompaña al ser humano desde su nacimiento, evoluciona y se perfecciona a lo largo de la vida. La voz es el componente sonoro de la comunicación que sustenta la realización efectiva del lenguaje verbal; está presente en todos los actos de habla de los individuos y por lo tanto, desde temprana edad se percibe como algo innato y que siempre vamos a tener.

Rara vez pensamos que la voz, por cualquier circunstancia puede faltar o se puede alterar y dar origen a disfunciones en la expresión verbal que hacen menos efectiva la comunicación. La reflexión anterior debería ser válida para todo ser humano, pero no es así; en nuestra cultura no se da importancia al uso correcto de la voz y se desconocen los cuidados para conservarla. Esta situación, sin ser la ideal, no trae consecuencias negativas para la salud de la población en general, pero sí compromete la capacidad comunicativa interpersonal. En los individuos que usan la voz para el desempeño de un trabajo, como es el caso de los docentes, es diferente; el uso incorrecto de la técnica vocal origina problemas en el bienestar físico e interfiere en el desempeño correcto de la profesión, no permite la proyección vocal efectiva, fundamento esencial de la comunicación en público

Fonoaudióloga con especialización en Docencia Universitaria, Profesora Institución Universitaria Fundación Escuela Colombiana de Rehabilitación 


\section{¿QUÉ ES LA VOZ?}

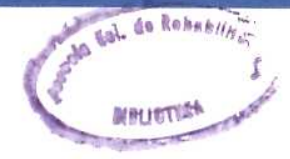

Para muchas personas la voz es el sonido producido en los pliegues vocales por el paso del aire; definición bastante simple que se refiere sólo a un componente de la voz. La voz debe ser entendida según Bustos (1995) como: "...una manifestación expresiva de la persona en su totalidad, a través de ella cada individuo logra expresarse y comunicarse con sus semejantes de una manera singular y única. La voz trasluce la vida psíquica y emocional de quien se expresa y en ella subyace una compleja acción de nervios, huesos, cartílagos y músculos, que implican al cuerpo de manera global" (p.25).

Sobre la base del concepto anterior podemos deducir la importancia de la voz en la vida de una persona y en su comunicación; no sólo porque es el soporte del lenguaje oral, sino por ser la tarjeta de presentación de cada individuo y en la cual se reflejan muchos aspectos de su personalidad.

\section{RIESGOS DEL DOCENTE}

Los riesgos que potencialmente pueden desencadenar desórdenes de la voz en los docentes, pueden llevar a perturbaciones en la salud, interferencia en la realización de algunas funciones y compromiso de su bienestar psicológico y social.

Con base en la clasificación de riesgos descrita por Álvarez, R. y Mancera, M., (1991) y la experiencia adquirida en el diagnóstico y la intervención de desórdenes de la voz, en este artículo se presenta el siguiente cuadro que resume las repercusiones sobre la voz de factores de riesgo en el ejercicio de la labor docente.

\begin{tabular}{|c|c|c|}
\hline RIESGO & AGENTE & REPERCUSIONES SOBAE LA VOZ \\
\hline FISICOS & Ruido & $\begin{array}{l}\text { Uso vocal en ambientes ruidosos. } \\
\text { Aumento de intensidad de la voz. } \\
\text { Esfuerzo vocal. } \\
\text { Mal uso vocal. } \\
\text { Ambientes con temperaturas extremas: } \\
\text { cambios en mucosa nasal y laríngea que comprometen } \\
\text { respiración, vibración cordal y resonancia. } \\
\text { Esfuerzo fonatorio. } \\
\text { Aumenta con componente alérgico. }\end{array}$ \\
\hline QUIMICOS & Material particulado & $\begin{array}{l}\text { Cambios en mucosa nasal y laríngea que comprometen } \\
\text { respiración, vibración cordal y resonancia: } \\
\text { Esfuerzo fonatorio, carraspeo frecuente, } \\
\text { sensación de resequedad. } \\
\text { Aumenta con componente alérgico. }\end{array}$ \\
\hline ERGONOMICOS & Posiciones forzadas & $\begin{array}{l}\text { Uso vocal con posturas inapropiadas: } \\
\text { Apoyo de voz en laringe. } \\
\text { Cambio en fisiología vocal. } \\
\text { Tipo respiratorio inapropiado. } \\
\text { Cansancio muscular y vocal. }\end{array}$ \\
\hline PSICOSOCIALES & $\begin{array}{l}\text { Estrés } \\
\text { Uso vocal prolongado } \\
\text { Deficiente técnica vocal }\end{array}$ & $\begin{array}{l}\text { Tensión Muscular. } \\
\text { Cansancio vocal. } \\
\text { Mal uso de la voz. } \\
\text { Esfuerzos fonatorios. } \\
\text { Pérdida de cualidades de la voz. }\end{array}$ \\
\hline
\end{tabular}


Las repercusiones de los riesgos anteriores se agravan por el desconocimiento que tienen los docentes sobre los cuidados de la voz, que implica el uso vocal excesivo durante procesos inflamatorios e infecciosos en órganos que intervienen en la producción de la voz, el abuso y mal uso vocal, la falta de una práctica cotidiana de calentamiento de la voz para el ejercicio docente, que se considera como un uso profesional de la voz.

El cuadro anterior resume algunos riesgos inherentes al ejercicio profesional del docente, pero en la prevención y control de los desórdenes de la voz no se debe pasar por alto, que los desórdenes de la voz tienen otras causas: neurológica, hormonal, digestiva, oncológica, auditiva, psicológica, músculo esquelética, anatómica, respiratoria, hábitos de habla inapropiados desde la infancia, hábitos nocivos (cigarrillo, alcohol.) Cualquiera de las anteriores puede estar presente y aumentar la probabilidad de desarrollar un desorden de la voz.

\section{SÍNTOMAS DE ALERTA Y CONSECUENCIAS}

En la población general la presencia esporádica de uno o varios síntomas que comprometen la función vocal no siempre es motivo de preocupación, pues como se describió en el párrafo anterior, las causas de los desórdenes de la voz son múltiples y variadas; generalmente cuando se tienen enfermedades infecciosas o virales que comprometen la vía aérea superior (nariz, faringe, laringe) pueden presentarse cambios en la voz. Por el contrario, en personas que utilizan la voz como herramienta de trabajo (profesionales de la voz) cualquier cambio en la voz sea pasajero o permanente requiere atención.

Los síntomas se pueden manifestar en varias formas: cambios en los pliegues vocales como enrojecimiento, inflamación, engrosamiento, lesiones (nódulos, pólipos, úlceras), los cuales se detectan en un examen realizado por el médico otorrinolaringólogo.

Cambios en el comportamiento vocal y respiratorio como: gesto respiratorio rígido, respiración superior, ausencia de soplo fonatorio, tensión en cuello, lengua, labios y mandíbula; exagerada contracción del velo del paladar, intensidad vocal aumentada o disminuída, alteraciones en el tono y timbre de la voz, pérdida de la verticalidad, incoordinación fono-respiratoria, bajo rendimiento respiratorio en función de la palabra. Estos se detectan en un examen de voz realizado por un fonoaudiólogo.

\section{SEÑALES DE ALERTA}

El individuo con algún desorden de la voz percibe algunos cambios durante el acto de hablar, estos pueden ser:

o Falta de eficacia en el acto vocal.

o Fatiga fonatoria (cansancio al hablar o después).

o Dolor y tensión en cuello y parte alta de la espalda.

o Opresión respiratoria (falta de aire).

o Carraspeo frecuente.

o Sensación de cuerpo extraño en la garganta.

La principal consecuencia de la falta de control sobre las señales de alerta es la aparición de un desorden de la voz (disfonía) que se define según la Asociación Americana de Habla- Lenguaje- Audición, y algunos criterios reportados por Francoise Le Huche (1993) como la producción anormal o ausencia de calidad, tono, intensidad, resonancia y duración de la voz; uso inapropiado para la edad, sexo y ocupación del individuo. El desorden puede ser momentáneo o persistente y percibido por la persona que la presenta o por su entorno.

En un docente la presencia de uno o varios síntomas de alteración vocal, debe ser motivo de preocupación y conducir a un control oportuno de los mismos. La situación ideal para los docentes sería que nunca se presentaran signos de molestia vocal, muchos de ellos ligados al abuso y mal uso de la voz. Esto se podría lograr si todos los profesores en ejercicio activo de su profesión manejaran una correcta técnica vocal.

\section{PREVENCIÓN Y CONTROL DE DESÓRDENES DE LA VOZ}

La prevención de los desórdenes de la voz en los docentes se debe enfocar hacia la realización de diversas acciones ejecutadas como ya se dijo anteriormente, por los docentes, las personas o instituciones responsables de su formación, empleadores y servicios de salud. A continuación se describen algunas acciones dirigidas al control y prevención de desórdenes de la voz.

O Incluir en los planes de estudio de los futuros docentes asignaturas que lleven al conocimiento de la fisiología vocal y su proyección efectiva.

o Planear evaluaciones periódicas de la voz, para detectar oportunamente desórdenes de la misma.

o Realizar campañas de promoción que proporcionen información y educación sobre los cuidados de la voz y su uso correcto. Esto ayudará a crear conciencia 
en los docentes de la responsabilidad que tienen sobre la conservación de su voz y mejorará el conocimiento sobre las consecuencias de su uso inapropiado.

o Diseñar programas de formación donde se desarrollen habilidades en el uso vocal.

o Ubicar las aulas de clase en zonas de poco ruido, si esto no es posible se debe realizar atenuación del ruido exterior. El desarrollo en los docentes de habilidades de control audio fonatorio, podrá minimizar el esfuerzo vocal que se presenta al hablar en ambientes ruidosos.

o Diseñar aulas de clase con ventilación apropiada y por parte del docente tomar medidas que prevengan los efectos de los cambios bruscos de temperatura.

o Implementar el uso de tableros acrílicos para evitar el uso de tiza que puede ser perjudicial especialmente para docentes que tienen problemas alérgicos. En caso de ser necesario el uso de tiza, se recomienda al docente borrar el tablero en forma correcta para disminuir la dispersión del polvo.

- Colocar los tableros a una altura apropiada, de manera que cuando el docente escriba y hable en forma simultánea no genere posiciones forzadas con su cuerpo que repercutan en el correcto funcionamiento del aparato fonador.

o Controlar el uso vocal prolongado. Toda actividad física realizada con el cuerpo humano en forma excesiva y sin métodos apropiados lleva a la fatiga, la voz no es la excepción; su uso sin control y por un tiempo excesivo lleva a cansancio vocal y se manifiesta en la pérdida de las cualidades de la voz. Por tanto es responsabilidad de los docentes comprender que el uso de la voz profesional conlleva moderación en su uso y hace necesario la realización de ejercicios de calentamiento vocal, previos al uso de la voz por tiempo prolongado.

\section{INTERVENCIÓN DIRECTA}

La producción de la voz requiere interacción dinámica entre varios componentes anatómicos y fisiológicos relacionados con la respiración, la vibración cordal, la resonancia y la articulación. El uso correcto de la voz se tiene, si se cumple con las siguientes condiciones:

o Postura y control muscular del cuerpo, cuello y cara.

o Técnica respiratoria que lleve a un soplo fonatorio óptimo.

o Correcta vibración de pliegues vocales y flexibilidad en los movimientos de la laringe.

o Coordinación de la respiración con la vibración de los pliegues vocales.

o Acople de los resonadores.

o Flexibilidad en tono e intensidad de la voz.

o Claridad en la producción de las palabras.
El uso profesional de la voz, como en el caso de los profesores, requiere de un entrenamiento para desarrollar habilidades. Al igual que un deportista debe entrenarse físicamente para un buen desempeño en competencias y un cantante profesional debe formarse en el dominio de la voz cantada, un profesor debe desarrollar habilidades en el uso correcto de la técnica vocal para voz hablada y debe realizar una rutina de ejercicios que lleven al buen empleo y uso cómodo de la voz, y así evitar desórdenes vocales que pueden llegar a ser crónicos.

\section{RECOMENDACIONES PARA EL CUIDADO DE LA VOZ}

o Evite carraspear y toser habitualmente.

o No grite habitualmente.

O Evite hablar en ambientes ruidosos.

o Al usar la voz en espacios abiertos o con grandes audiencias,use amplificación apropiada.

o Cante solamente dentro de la gama vocal en que se sienta cómodo.

o Controle la energía de proyección vocal para hablar en público.

o No hable mucho durante la realización de ejercicio físico agotador.

o Evite usar voz monótona de tono bajo.

o No contenga la respiración mientras piensa lo que va a decir.

o No hable con frases más largas que el ciclo respiratorio habitual.

o Manténgase relajado al hablar: cuerpo, mandíbula, lengua.

o No le exija a su voz más de lo que le exigiría al resto de su cuerpo.

o No utilice en exceso su voz cuando esté enfermo o cansado

o No utilice su voz cuando la sienta tensa

o No pase por alto los síntomas y reconozca los signos de alerta.

o Consulte cualquier cambio que sienta en su voz

o No se exponga a una contaminación excesiva o productos deshidratantes.

o Mantenga una buena postura.

El primer paso para usar la voz efectivamente y mantenerla sana, es la motivación para convertir los cuidados de la voz y la realización de los ejercicios en una rutina diaria y en una forma de vida.

Cuando haya logrado esto podrá conservar la voz y usarla efectivamente en forma permanente. 


\section{CONCLUSIONES}

El docente es un profesional de la voz y como tal, debe tomar conciencia de que las alteraciones de la voz no sólo repercuten en su capacidad comunicativa interpersonal, sino en el desempeño satisfactorio de su profesión.

Aunque las causas de desórdenes de la voz son múltiples y variadas, en los docentes un alto porcentaje son producidas por abuso y mal uso de la voz originando lo que se conoce como fono trauma. El docente debe comprometerse activamente con la prevención y control de las disfunciones vocales para tener un óptimo bienestar físico, mental y social.

Es necesario que tanto especialistas en salud ocupacional, como médicos e higienistas, al igual que profesionales de otras disciplinas que de una u otra forma tienen que ver con el estudio de la voz humana como fonoaudiólogos y otorrinolaringólogos, se preocupen por la promoción y prevención de la voz normal y sus desórdenes.

\section{BIBLIOGRAFIA}

ALVAREZ, Ricardo y MANCERA, Mario. Salud ocupacional. Manual guía. Bogotá: Ministerio de Trabajo y Seguridad Social. Arte Impreso, 1991

BUSTOS, Inés. Tratamiento de los problemas de la voz. Madrid : Ciencias de la educación preescolar y especial, 1995

LE HUCHE, Francoise y ALLALI, André. Patología vocal: semiología y disfonías funcionales. Barcelona : Masson S.S, 1993

MORALES, Patricia y LASTRA, Clara. Patología vocal en la Caja Nacional de Previsión. en : Revista Mensaje. No 9 (julio-agosto 1985)

MORRISON, Murria y RAMAJE, Linda. Tratamiento de los trastornos de la voz. Barcelona : Masson, 1996 AMERICAN SPEECH-LANGUAGE-HEARIN ASSOCIATION. Definitions of communication disorders and variations. En Asha 35. suppl.10.

LONG, Joanne et al. Voice problems and risk factors among aerobics Instructor. En Jorunal of voice. Vol 12. No 2,1998

MATTISKE, Jacqueline et al. Vocal problems among teachers: a review of prevalence, causes, prevention, and treatment. En : Journal of voice. Vol 12. No 4, 1988 